\title{
Análise de Produções da Revista Brasileira de Orientação Profissional
}

\author{
Ana Paula Porto Noronha \\ Cristiane Deantonio Ventura \\ Dario Cecilio-Fernandes \\ Juliana Carnevalli Siqueira Nery \\ Juliana Maximila de Paula Bueno \\ Luana Luca \\ Ivan Baroncelli Neto \\ Universidade São Francisco \\ São Paulo, SP, Brasil \\ Maria Áurea Pereira Silva \\ Pontifícia Universidade Católica de Campinas \\ Campinas, SP, Brasil
}

\begin{abstract}
RESUMO
Investigar a produção científica de determinada área de conhecimento permite promover o desenvolvimento organizado dela. Esse estudo analisou quanti e qualitativamente as publicações da Revista Brasileira de Orientação Profissional, de 2007-2011. Foram arrolados 68 artigos, dos quais 53 empíricos e 15 teóricos. Para a análise das temáticas, houve a organização em categorias, sendo que a relação entre construtos e variáveis foi a que agregou o maior número de artigos. Os adolescentes foram os mais pesquisados. Houve grande diversidade de instrumentos utilizados nas investigações; a técnica mais utilizada foi a entrevista. Dentre as referências citadas nos artigos, foram mais mencionadas aquelas publicadas a partir de 2000, e de modo geral, as estrangeiras foram mais citadas. São discutidas implicações para pesquisas futuras.
\end{abstract}

Palavras-chave: Cientometria. Orientação profissional. Produção científica.

\section{ABSTRACT}

Analysis of publication of Revista Brasileira de Orientação Profissional

Investigate the production of a particular area of scientific knowledge allows her to promote the development organized it. This study analyzed the publications of the Revista Brasileira de Orientação Profissional during the 2007 to 2011 period. Were analyzed 68 articles, witch 53 empirical and 15 theoretical. For analysis of the themes, there were category organizations, so that the relationship between the contextual variables and constructs was added to the largest number of articles. Besides that, the researcher focus more on teenagers, they apply different instruments, and the most used technique was the interview. Regards to reference, the articles cited that was published since 2000 and the majority was international. Besides that it were discussed implications for future research.

Keywords: Bibliometrics. Vocational guidance. Scientific production.

\section{RESUMO}

Análisis de las Producciones de la Revista Brasileira de Orientação Profissional

Investigar la producción científica de un área particular del conocimiento permite promover su desarrollo ordenado. Este estudio analizó cuantitativa y cualitativamente las publicaciones de la Revista Brasileña de Orientación Profesional, de 2007-2011. Fueron evaluados 68 artículos, de los cuales 53 empíricos y 15 teóricos. Para el análisis de las temáticas, hubo una organización en categorías, siendo que la relación entre los constructos y las variables fue la que se añadió al mayor número de artículos. Los adolescentes fueron los más estudiados. Hubo gran diversidad de instrumentos utilizados en las investigaciones; la técnica más utilizada fue la entrevista. Entre las referencias citadas en los artículos, fueron más mencionadas aquellas publicadas a partir del año 2000, y en general, los extranjeros fueron los más citados. Por fin, se discuten las implicaciones para las investigaciones futuras.

Palabras clave: Cienciometría. Orientación profesional. Producción científica. 


\section{INTRODUÇÃO}

A investigação das produções científicas é necessária, uma vez que ela pode fornecer indicadores de determinada área de conhecimento, de modo a assinalar os incrementos a serem efetivados. Adicionalmente, a análise merece a atenção de pesquisadores, pois por intermédio dela é possível identificar elementos relevantes como a profundidade, amplitude e tendência, a carência em determinadas áreas, quem e como se publica, além de orientar o pesquisador em novas buscas (Melo-Silva, Leal \& Fracalozzi, 2010; Witter, 1999).

Noronha e Ambiel (2006) e Melo-Silva, Leal e Fracalozzi (2010), ao analisarem a produção na área de Orientação Profissional (OP), enfatizaram que os resultados advindos dos estudos podem fomentar o desenvolvimento da área e denunciar a possível desproporcionalidade de publicações em alguns conteúdos em detrimento de outros. Para verificar a produção científica é necessária a sistematização das publicações e a utilização de método, tal como preconizado por Bufrem e Prates (2005). Para Witter (1999), a bibliometria é definida como um processo de análise quali e quantitativa da produção, divulgação e uso da informação. Os métodos utilizados constituemse em instrumentos que subsidiam estudos relativos à revisão bibliográfica.

Essa pesquisa versa sobre a análise de uma revista temática de OP e, outros artigos foram realizados com objetivos semelhantes (Ivatiuk e Yoshida, 2010; Noronha e cols., 2006; Teixeira, Lassance, Silva e Bardagi, 2007; dentre outros). A escrita e a publicação são indispensáveis na construção do conhecimento científico, assim, as investigações devem ser propagadas, tornando-se acessível à comunidade (Sampaio, 2008). Nesse contexto, a publicação é um compromisso dos pesquisadores de modo que por seu intermédio há a difusão do conhecimento, possibilitando dessa forma, a comunicação sobre o que foi obtido, o intercâmbio e as experiências do estudo (Sampaio, 2008; Sabadini, Sampaio e Koller, 2009).

Levenfus (2010) descreve a Orientação Profissional como um processo que informa e orienta sobre variáveis importantes a respeito do mercado de trabalho, bem como sobre questões do orientando, com o intuito de criar condições para que as pessoas se conheçam melhor, percebam suas identificações, adquiram melhores condições de organizar seus projetos de vida, e consequentemente, façam escolhas profissionais oportunas. Em complemento, Noronha, Santos e Sisto (2010) afirmam que a OP oferece condições de reflexão que pretendem facilitar a busca da identidade profissional. Para acompanhar o cenário de constantes mudanças na concepção de mundo, de trabalho e de sua relação com o homem, o campo da Orientação Profissional, segundo Lehman (2010), passa a ter como foco uma problemática mais ampla, indo além da escolha profissional. A OP surge como um processo necessário em diversas etapas da vida nas quais podem ocorrer rupturas na trajetória pessoal e profissional.

A área da Orientação Profissional vem passando por transformações, principalmente, no cenário nacional, no que se refere à produção científica na área. Pesquisas recentes vêm demonstrando essas mudanças e evidenciando que há uma preocupação por parte dos pesquisadores da área pela obtenção de dados empíricos (Melo-Silva e cols., 2010; Noronha e Ambiel, 2006; Teixeira e cols., 2007; entre outros). A seguir, serão apresentados trabalhos de análise de produção científica na área.

Sob esta perspectiva, Noronha e Ambiel (2006) verificaram aumento da divulgação de artigos teóricos e de pesquisa na área de OP a partir da década de 1990. $\mathrm{O}$ estudo analisou bases de dados eletrônicos, e foram encontradas publicações desde 1950 até a data da pesquisa, totalizando 191 artigos. Predominantemente foram encontrados revisões teóricas e aqueles trabalhos que expressavam a preocupação com a qualidade dos instrumentos de avaliação.

O exame da produção de teses e dissertações relacionadas à área de orientação profissional e vocacional foi o objetivo de Noronha e cols. (2006). Os autores fizeram uma busca em bases de dados eletrônicas da Coordenação de Aperfeiçoamento de Pessoal de Nível Superior (CAPES) e da Biblioteca Virtual da Saúde (BVS-Psi) no período entre 1969 e 2005. Foram identificados 100 trabalhos, sendo que os resultados indicaram o crescimento da área de OP a partir da década de 1990, revelando também uma preponderância dos relatos de pesquisa ou de experiência prática. Os autores consideraram esse achado positivo para a área, com vistas a possibilitar a estruturação de um corpo científico e teórico voltado para o contexto nacional.

De maneira similar ao presente estudo, Teixeira e cols. (2007), realizaram um mapeamento dos artigos publicados na Revista da Associação Brasileira de Orientação Profissional (ABOP) e Revista Brasileira de Orientação Profissional, no período de 1997 a 2006. Foram analisados 85 artigos de forma qualitativa e quantitativa. Os principais resultados revelaram o aumento de estudos empíricos em relação aos teóricos; predomínio de pesquisas realizadas com discentes do ensino médio; estudos voltados para o desenvolvimento de carreira de universitários; realização de pesquisas 
com populações em situação de vulnerabilidade; escassez quanto à parceria interinstitucional entre pesquisadores; convergência de publicações em poucos centros de pesquisas e com predominância dos Estados de São Paulo, Rio Grande do Sul e Santa Catarina. Para os autores essa ampliação dos grupos investigados para além do adolescente que pretende ingressar na universidade, permite trabalhar não só com a transição escola-trabalho, mas com outros elementos do processo de desenvolvimento de carreira, nem sempre privilegiados no Brasil. Por fim, os autores chamaram a atenção para a diversidade de temas estudados e para a pequena colaboração entre pesquisadores e diferentes centros de pesquisa.

Outra análise de produção científica foi realizada por Rueda (2009), que avaliou a produção da Revista Brasileira de Orientação Profissional, de 2003 a 2008. Dos 81 artigos encontrados, houve predominância de relatos de pesquisas; maior publicação da região Sudeste e Sul; alta frequência de múltiplos autores e, na sua maioria, do sexo feminino. Ademais, foi utilizado grande número de testes, inventários e escalas e no que concerne às referências, ao mesmo tempo em que a maioria dos artigos se baseou em livros ou capítulos de livros, uma menor parte, em artigos de pesquisa.

Melo-Silva e cols., (2010) objetivaram analisar os trabalhos publicados em congressos brasileiros de orientação vocacional e profissional no período 1999 a 2009. Foram selecionados 733 resumos relativos às mesas redondas, apresentações orais e pôsteres de seis eventos ocorridos no referido período. Os principais resultados mostraram a preponderância de comunicações orais, relatos de pesquisa e de experiências, com adolescentes e adultos jovens que pretendem ingressar no curso superior. Predominou a publicação individual, seguida de estudos em coautoria (dois ou mais autores) de pesquisadores das regiões Sudeste e Sul. Na sua maioria os estudos foram sobre escolha profissional, intervenção, serviços e instrumentos. A temática referente às transições no ciclo vital e orientações a distintas populações em condições socioeconômicas desfavorecidas também esteve presente. Por fim, os autores evidenciaram o aumento do número de trabalhos apresentados, exceto no ano de 2005, além da maior participação de orientadores e pesquisadores de outros países.

Com o objetivo de realizar uma revisão da literatura publicada na última década sobre orientação profissional de pessoas com deficiências, Ivatiuk e Yoshida (2010) encontraram 55 referências (nacionais e estrangeiras) divididas entre artigos, teses e dissertações, capítulos de livros e livros. Os resultados apontaram para um maior número de estudos de natureza empírica. Grande parte dos estudos não evidenciou o modelo teórico utilizado e, ao lado disso, os processos de intervenção foram na sua maioria de forma individual. Os autores concluíram que há um número relativamente baixo de trabalhos nacionais e internacionais no que diz respeito às práticas em OP de pessoas com algum tipo de deficiência.

Em síntese, no que concerne aos estudos nacionais, pode-se observar que houve um consenso quanto à importância do processo de Orientação Profissional aplicado a diferentes grupos, além de análise das teorias utilizadas, bem como das intervenções. Ademais, destaca-se o visível desenvolvimento de publicações na área.

No que refere ao âmbito internacional, é habitual revisões dessa modalidade. A título de exemplo, cita-se a pesquisa de López e cols. (2010), sobre a produção acadêmica de Psicologia a partir dos artigos publicados entre 2005 e 2007 em revistas indexadas pela PsicoRedalyc. Os principais resultados mostraram que três em cada quatro artigos são escritos em colaboração de pesquisadores da mesma instituição; que houve pouca participação de autores estrangeiros na maioria dos artigos e $80,9 \%$ dos autores tiveram a proporção de uma publicação a cada três anos. Os pesquisadores sugeriram o fortalecimento e o estímulo de publicações em periódicos de outros países.

Arbona (2000) revisou a literatura publicada em 1999 sobre o desenvolvimento e aconselhamento de carreira, por intermédio da $P$ sycINFO computadorizada, livros, capítulos de livros e artigos. A discussão foi sistematizada em três grandes áreas, a saber, publicações relacionadas a abordagens teóricas, novos instrumentos de avaliação, e intervenções de carreira e programas. Os resultados revelaram que grande proporção de publicações foi baseada em uma abordagem teórica. Ao lado disso, foram encontrados estudos de validade dos instrumentos existentes e desenvolvimento de novos instrumentos para avaliar construtos emergentes. Quanto à amostra houve mais estudos com mulheres, minorias étnicas e raciais e pessoas com deficiência. Além disso, os pesquisadores examinaram fatores que podem ser importantes para a compreensão da evolução na carreira de várias populações, envolvendo a aculturação, identidade étnica, discriminação e a experiência de fazer parte de um grupo minoritário no contexto de ambientes educacionais e de trabalho.

Por fim, Guindon e Richmond (2005) estudaram as publicações de 2004 sobre desenvolvimento e aconselhamento de carreira, em alguns periódicos da área, como o The Carrer Development Quartely, Journal of Vocational Behavior, Journal of Career Development, Journal of Career Assessment e Journal 
of Emplyment Counseling. Os autores analisaram 173 artigos e enfatizaram que os trabalhos, de maneira geral, expressavam uma visão moderna do aconselhamento de carreira, da qual fazem parte a mudança na economia global; as transições escola para a faculdade; da faculdade para o trabalho, ou mesmo do emprego à escola novamente; e os diferentes clientes de desenvolvimento de carreira.

Desse modo, torna-se relevante que estudos dessa natureza sejam realizados, a fim de acompanhar o desenvolvimento da Orientação Profissional como um dos campos de aplicabilidade da psicologia. Portanto, o objetivo do presente estudo é analisar as produções científicas realizadas no período de 2007 a 2011 na Revista Brasileira de Orientação Profissional.

\section{MÉTODO}

\section{Material}

Foi realizada uma revisão por meio da análise documental de artigos científicos da Revista Brasileira de Orientação Profissional. Para o presente estudo foram selecionadas as publicações do período de 2007 a 2011 disponíveis online no endereço www. bvs-psi.org.br, com foco nos artigos. Foram avaliados cinco volumes, nove números, sendo que no ano de 2011 considerou-se apenas o primeiro. Assim, foram encontrados 68 artigos agrupados em empíricos $(\mathrm{N}=53)$ e teóricos $(\mathrm{N}=15)$.

\section{Procedimentos e análise dos dados}

Os dados foram analisados quantitativa e qualitativamente. Os resultados quantitativos foram verificados por meio da estatística descritiva, sendo analisado o número de autores, seus respectivos estados, bem como a filiação institucional deles. Assim como, diferenciou-se a pesquisa teórica da empírica. Em outro momento, realizou-se a categorização do modelo teórico, da temática do artigo, quantos instrumentos e técnicas foram utilizados e categorização deles. Por último, foram analisadas as referências nos artigos.

Em relação à análise qualitativa, a fim de estudar a temática dos artigos foram definidas categorias de análise, tal como preconizado por Bardin (1977). A seis categorias eram excludentes e são descritas abaixo.

Em 'abordagens e modelos teóricos' foram incluídos aqueles artigos que se propuseram a discutir a Orientação Profissional sob a perspectiva de uma determinada visão de homem; como exemplo, citase orientação vocacional com base na abordagem dinâmica. O 'foco no instrumento/na técnica' englobou os estudos que tiveram como tônica central o instrumento ou a técnica. A título de exemplo, cita-se buscar evidências de validade convergente entre dois instrumentos (Teste de Fotos de Profissões-BBT-Br e Self Directed Search $-C E$ ). Por 'análise da produção científica compreendeu-se publicações que investigavam o estado da arte (analisar a produção científica da Revista Brasileira de Orientação Profissional).

A quarta categoria intitulada 'análise de resultados e processos' reuniu estudos que verificaram eficácia de processos de OP (folow up), como avaliar um serviço de $O P$ de um Centro de Psicologia Aplicada. As relações entre construtos afins e outras variáveis estiveram contempladas na quinta categoria. Nela foram organizados os trabalhos que promoveram comparações entre construtos e instrumentos, sem o compromisso de estudá-los psicometricamente (levantar dúvidas $e$ interesses de adolescentes em relação à escolha $e$ avaliar o desenho da figura humana). Por fim, em 'outros' foram alocados os artigos que não puderam ser incluídos nas anteriormente descritas.

Utilizou-se o mesmo procedimento para verificar o objetivo com o qual técnica/instrumento foram usados no artigo. Neste caso, considerou-se apenas os artigos que utilizaram técnica ou instrumento de coleta de dados, totalizando 45 unidades, pois não estiveram incluídos os teóricos. Foram definidas cinco categorias, quais sejam, identificação de variáveis (construto ou contextos) relacionados ao processo de OP ou ao momento de escolha; levantamento de dados com o objetivo de caracterização da amostra; estudo de parâmetros psicométricos dos instrumentos; relação entre instrumentos sem o objetivo de estudar qualidades psicométricas; e outros. Como exemplo da primeira categoria, apresenta-se analisar o papel dos estágios na transição para o trabalho. Por levantamento de dados entendeu-se comparar desempenhos de adolescentes e adultos no BBT. Já estudar a validade convergente foi inserida na categoria estudo de parâmetros psicométricos. Por fim, analisar a relação entre Escala de Aconselhamento Profissional (EAP) e SDS é um exemplo da quarta categoria de análise. Em outros, foram inseridos artigos que não se relacionavam com a categorização determinada.

As categorias foram submetidas à apreciação de cinco juízes independentes, sendo alunos de mestrado de um programa de pós-graduação em Psicologia, além de uma doutora, que avaliaram os artigos. Para a primeira categorização (temática), houve de 75 a 100\% de concordância em 35 dos artigos. Em relação à segunda categorização, qual seja, objetivo do uso da técnica, a concordância de 75 a 100\% ocorreu em 52 dos 66 artigos. Nos momentos em que o consenso não foi obtido, quem decidiu sobre a categorização final foi a juíza com maior titulação. 


\section{RESULTADOS}

Os resultados serão apresentados na mesma sequência em que foram comentados no método, ou seja, primeiro os dados quantitativos, e em seguida, os qualitativos. Na Tabela 1 observaram-se as frequências relativas ao número de autores, tal como a respectiva porcentagem.

TABELA 1

Frequências relativas ao número de autores nos artigos

\begin{tabular}{crrc}
\hline Autores & $N$ & $\%$ & $\%$ acumulado \\
\hline 1 & 15 & 22,1 & 22,1 \\
2 & 34 & 50,0 & 72,1 \\
3 & 11 & 16,2 & 88,2 \\
4 & 5 & 7,4 & 95,6 \\
5 & 2 & 2,9 & 98,5 \\
6 & 1 & 1,5 & 100,0 \\
Total & 68 & 100,0 & \\
\hline
\end{tabular}

Em observação à Tabela 1, identificou-se que houve uma concentração na publicação com até três autores, apresentando uma porcentagem acumulada de $88,2 \%$, dividida em 34 (50\%) artigos com dois autores, 15 $(22,1 \%)$ com um autor e $11(16,2 \%)$ com três autores. Além disso, a freqüência de menor ocorrência foi com seis autores. Nos resultados apresentados na Tabela 2 verificou-se a participação de diferentes autores por unidades federativas (Estados) e instituições.

TABELA 2

Autores e universidades por estado/países

\begin{tabular}{lcrrrr}
\hline \multicolumn{1}{c}{ Países } & Estados & Autores & \multicolumn{1}{c}{$\%$} & Universidades & \multicolumn{1}{c}{$\%$} \\
\hline Brasil & SP & 47 & 30,91 & 5 & 13,88 \\
& MG & 19 & 12,50 & 10 & 27,77 \\
& BA & 1 & 0,66 & 1 & 2,78 \\
& PR & 4 & 2,63 & 1 & 2,78 \\
& DF & 4 & 2,63 & 1 & 2,78 \\
& RS & 21 & 13,82 & 3 & 8,33 \\
& ES & 2 & 1,32 & 1 & 2,78 \\
& SC & 4 & 2,63 & 1 & 2,78 \\
& RJ & 1 & 0,66 & 1 & 2,78 \\
& MA & 1 & 0,66 & 1 & 2,78 \\
Portugal & & 43 & 28,28 & 6 & 16,66 \\
Cuba & & 2 & 1,32 & 2 & 5,56 \\
Canadá & & 1 & 0,66 & 1 & 2,78 \\
Venezuela & & 1 & 0,66 & 1 & 2,78 \\
China & & 1 & 0,66 & 1 & 2,78 \\
Total & & 152 & 100,00 & 36 & 100,00 \\
\hline
\end{tabular}

Nota-se, no âmbito nacional, o predomínio de autores em São Paulo, Minas Gerais e Rio Grande do Sul, revelando uma elevada produção dos trabalhos científicos nesses estados, tendo São Paulo uma maior concentração de autores. No contexto internacional, dentre os citados, Portugal detém maior representatividade. Os dados revelam pequena parceria entre os pesquisadores inter-países.

Ainda na Tabela 2, há informações quanto às Universidades dos autores. Considerou-se o número de instituições diferentes dentro de cada Estado e não a quantidade de vezes que cada instituição aparecia, ou seja, quando uma universidade aparecia diversas vezes dentro de um Estado, esta era computada uma única vez. Os resultados indicam que o maior número de universidades distintas foi no Estado de Minas Gerais, totalizando $10(27,77 \%)$ universidades. No exterior, o número de instituições de ensino envolvidas é maior em Portugal com $6(16,66 \%)$.

Em relação ao tipo de manuscrito, verificou-se que dos 68 artigos analisados, 53 (77,9\%) eram empíricos e $15(22,1 \%)$ teóricos. Do total, apenas 41,2\% $(\mathrm{N}=28)$ explicitaram o modelo teórico utilizado no estudo, sendo que houve repetição dos modelos sócio histórico $(\mathrm{N}=2)$, sócio cognitivo de Bandura $(\mathrm{N}=2)$ e de autoeficácia $(\mathrm{N}=3)$. Os demais modelos teóricos foram de títulos distintos, como por exemplo, a teoria de Holland, desenvolvimento de carreira, abordagem sistêmica, entre outros. Na análise dos artigos pode-se observar que os autores não deixam explícita a teoria utilizada na pesquisa e na maior parte deles falta a definição da mesma.

Quanto à caracterização da amostra foram analisados 53 artigos, desses 22 (41,5\%) foram realizados com adolescentes, sendo nesse estudo considerado como sujeitos cursando até o ensino médio, 20 (37,7\%) com adultos, esses maiores de idade, trabalhando ou cursando nível superior, quatro $(7,6 \%)$ mistos, ou seja, participaram nos estudos tanto adultos como adolescentes, e sete $(13,2 \%)$ não se enquadram em nenhuma das categorias. Posteriormente, os artigos foram categorizados por temática, tal como explicitado na Tabela 3.

TABELA 3

Frequência relativas às categorias temáticas

\begin{tabular}{lrr}
\hline \multicolumn{1}{c}{ Categorias Temáticas } & $N$ & $\%$ \\
\hline Abordagem teórica & 13 & 19,1 \\
Foco no instrumento/ técnica & 7 & 10,3 \\
Análise da produção científica & 4 & 5,9 \\
Análise de resultados e processo & 6 & 8,8 \\
Relações entre construtos afins e & 32 & 47,1 \\
outras variáveis & & \\
Outros & 6 & 8,8 \\
Total & 68 & 100,0 \\
\hline
\end{tabular}


Os resultados obtidos apontam uma concentração maior na categoria Relações entre construtos afins e outras variáveis $(47,1 \%)$, na qual foram reunidos os trabalhos que estudaram as associações entre diferentes conceitos teóricos. Já a Análise de produção científica foi a temática menos presente nos artigos estudados $(5,9 \%)$.

No que diz respeito ao número de instrumentos utilizados nos artigos, em 47,1\% (N=32) não houve aplicação de nenhum; em 29,4\% ( $\mathrm{N}=20)$ um instrumento foi utilizado; $14,7 \%(\mathrm{~N}=10)$ contaram com dois; $2,9 \%$ $(\mathrm{N}=2)$ com três e em 5,9\% $(\mathrm{N}=4)$ foram computados o uso de quatro. Convém destacar que nesta categoria foram incluídos inventários, escalas, questionários, testes psicológicos, dentre outros. Com o intuito de aprofundar a informação, fez-se o levantamento dos instrumentos mais utilizados. O dado pode ser melhor visualizado na Tabela 4.

TABELA 4

Instrumentos utilizados nos artigos publicados na revista $A B O P$ de 2007 a 2011

\begin{tabular}{|c|c|c|}
\hline Instrumentos & $N$ & $\%$ \\
\hline $\begin{array}{l}\text { A Internet e a Pesquisa de Informação Escolar } \\
\text { e Profissional }\end{array}$ & 1 & 1,6 \\
\hline Abertura à Experiência (EFA) & 1 & 1,6 \\
\hline BBT & 3 & 4,7 \\
\hline $\begin{array}{l}\text { Career Decison-Making Difficulties } \\
\text { Questionnaire (CDDQ) }\end{array}$ & 1 & 1,6 \\
\hline Career Exploration Survey (CES) & 1 & 1,6 \\
\hline Checklist & 1 & 1,6 \\
\hline Desempenho acadêmico & 3 & 4,7 \\
\hline Desenhos de Profissionais com Estórias & 1 & 1,6 \\
\hline Escala de Aconselhamento Profissional (EAP) & 1 & 1,6 \\
\hline Escala de Atribuições de Carreira (EAC) & 1 & 1,6 \\
\hline Escala de Autoeficácia & 6 & 9,4 \\
\hline Escala de Comprometimento Organizacional & 1 & 1,6 \\
\hline Escala de entrincheiramento na carreira & 1 & 1,6 \\
\hline Escala de Exploração Vocacional & 2 & 3,1 \\
\hline Escala de Indecisão Vocacional & 1 & 1,6 \\
\hline Escala de Locus de Controle & 1 & 1,6 \\
\hline Escala de Maturidade & 2 & 3,1 \\
\hline Escala de satisfação com o curso de graduação & 2 & 3,1 \\
\hline Focus Group & 1 & 1,6 \\
\hline Formulário & 2 & 3,1 \\
\hline General Health Questionnaire-12 - GHQ-12 & 1 & 1,6 \\
\hline Inventário de Âncoras de Carreira & 1 & 1,6 \\
\hline $\begin{array}{l}\text { Inventário de Desenvolvimento Vocacional } \\
\text { para Estudantes (IDVE-FV) }\end{array}$ & 1 & 1,6 \\
\hline $\begin{array}{l}\text { Inventário Tipológico de Interesses } \\
\text { Profissionais (ITIP-96) }\end{array}$ & 1 & 1,6 \\
\hline $\begin{array}{l}\text { Latent and Manifest Benefi ts of Work - } \\
\text { LAMB-Scale }\end{array}$ & 1 & 1,6 \\
\hline Observação de Aula & 1 & 1,6 \\
\hline Personalidade & 4 & 6,3 \\
\hline Planejamento de Carreira & 1 & 1,6 \\
\hline Questionário & 18 & 28,1 \\
\hline Questionário de Busca Autodirigida (SDS) & 2 & 1,6 \\
\hline Total & 64 & 100,0 \\
\hline
\end{tabular}

Foram encontrados títulos diversos como, por exemplo, a Escala de Aconselhamento Profissional (EAP), Escalas Fatoriais de Neuroticismo (EFN), Escalas Fatoriais de Extroversão (EFEx), o Inventário de Âncoras de Carreira e o Teste de Fotos de Profissões (BBT-Br). Outro tipo de instrumento que se destacou foi o questionário com $29 \%$ de utilização, dentre os quais pode ser citado como exemplo, o Questionário de Autoeficácia Matemática (QAEM), além de questionários construídos pelos próprios pesquisadores.

As técnicas foram analisadas separadamente dos instrumentos, por possuírem concepções diferentes, tal como preconizado por Anastasi e Urbina (2000), Pasquali (2001), Urbina (2007). Em relação às técnicas, verificam-se nas pesquisas que $70,6 \%(\mathrm{~N}=48)$ dos estudos não utilizaram técnica, $17,6 \%$ (12) aplicaram apenas uma, $8,8 \%$ (6) usaram duas e $2,9 \%$ (2) três. As técnicas utilizadas nas pesquisas também foram consideradas no levantamento, como mostra a Tabela 5.

TABELA 5

Técnicas utilizadas nos artigos publicados na revista $A B O P$ de 2007 a 2011

\begin{tabular}{lrr}
\hline \multicolumn{1}{c}{ Técnicas } & $N$ & $\%$ \\
\hline Análise de documento & 1 & 3,6 \\
Análise do manual da disciplina de História & 1 & 3,6 \\
Entrevista & 6 & 21,3 \\
Entrevista semiestruturada & 11 & 39,1 \\
Entrevista telefônica & 1 & 3,6 \\
Evocação de metáforas & 1 & 3,6 \\
Focus Group & 1 & 3,6 \\
Fotografias tiras pelos participantes & 1 & 3,6 \\
Grupo de discussão & 1 & 3,6 \\
Investigação científica & 1 & 3,6 \\
Observação de aula & 1 & 3,6 \\
Produções textuais & 1 & 3,6 \\
Roteiro para análise de prontuários & 1 & 3,6 \\
Total & 28 & 100,0 \\
\hline
\end{tabular}

Percebe-se que a técnica que mais se destaca é a entrevista, sendo que a semiestruturada é a mais utilizada, seguida de entrevistas abertas. Em seguida, foram categorizados os objetivos com os quais as técnicas e os instrumentos foram usados nos artigos. Para tanto, 23 artigos não foram avaliados, pois não houve o registro de utilização, tal como apontado anteriormente. Na Tabela 6 encontraram-se os resultados relacionados às categorias apresentadas na seção procedimento (identificação de variáveis - construtos ou contextos - relacionados ao processo de $\mathrm{OP}$ ou ao momento de escolha; levantamento de dados com 
o objetivo de caracterização da amostra; estudo de parâmetros psicométricos dos instrumentos; relação entre instrumentos sem o objetivo de estudar qualidades psicométricas; e outros).

TABELA 6

Frequência dos objetivos das técnicas e instrumentos utilizados nos artigos

\begin{tabular}{lcr}
\hline \multicolumn{1}{c}{ Objetivo das técnicas e instrumentos } & $N$ & $\%$ \\
\hline $\begin{array}{l}\text { Identificação de variáveis relacionados ao } \\
\text { processo de OP }\end{array}$ & 13 & 28,9 \\
$\begin{array}{l}\text { Levantamento de dados ou caracterização da } \\
\text { amostra }\end{array}$ & 19 & 42,2 \\
$\begin{array}{l}\text { Estudo de parâmetro psicométrico dos } \\
\text { instrumentos }\end{array}$ & 4 & 8,9 \\
$\begin{array}{l}\text { Relação entre instrumentos, sem o objetivo de } \\
\text { estudar as qualidades psicométricas }\end{array}$ & 5 & 11,1 \\
$\begin{array}{l}\text { Outros } \\
\text { Total }\end{array}$ & 4 & 8,9 \\
\hline
\end{tabular}

De acordo com a Tabela 6, a categoria mais frequente foi o 'levantamento de dados ou caracterização da amostra' $(42,2 \%)$. Por levantamento de dados entendeu-se comparar desempenhos de adolescentes $e$ adultos no BBT. Em outra medida, a categoria menos presente foi 'estudos de parâmetros psicométricos dos instrumentos'.

Com relação à análise do levantamento das referências utilizadas nos artigos pesquisados, contabilizou-se 2.818 referências, sendo que 1.712 são estrangeiras e 1.116 nacionais, variando entre os anos de 1880 a 2010. Pode-se observar na Figura 1, que houve prevalência do uso de referências dos anos de 2000 a 2010, seguidos pela década de 1990 e 1980.

\section{DISCUSSÃO E CONSIDERAÇÕES FINAIS}

Dada a relevância da pesquisa em Psicologia Educacional, especificamente, em Orientação Profissional, realizou-se a análise quanti e qualitativa de publicações da Revista Brasileira de Orientação Profissional no período de 2007 a 2011. Este tipo de estudo possibilita a apresentação de um panorama do que, como e do quanto se tem pesquisado determinado assunto (Melo-Silva e cols., 2010; Witter, 1999).

Os resultados desta pesquisa remetem à elaboração de algumas considerações. Observou-se que a partir de 2007 houve certo aumento quanto à parceria de autores por publicação, principalmente em autorias do tipo dupla e tripla. Os trabalhos com quatro ou mais autores surgem mais frequentemente entre 2003 e 2006 (Teixeira e cols., 2007), mas no presente estudo constata-se que ainda são incipientes. A publicação de autoria única vem diminuindo de modo gradativo, se comparada ao período de 1997 a 2006. Assim, a produção científica individualizada do pesquisador brasileiro, demonstrada por Noronha e Ambiel (2006), paulatinamente, é substituída pela autoria múltipla.

No que concerne ao período e à quantidade de publicações, os resultados evidenciam aumento de publicações no referido periódico. Ao longo de dez anos (1997 a 2006) foram publicados 85 artigos, enquanto no período de quatros anos e um semestre (2007 a 2011) foram publicados 68 artigos, revelando ampliação de $80 \%$ de artigos em menos de meia década. Portanto, pode-se inferir que os pesquisadores têm publicado mais frequentemente em parcerias e, em maior quantidade. Ao lado disso, compreende-se que o aumento de produções revela o desenvolvimento da área no país (Sabadini, Sampaio e Koller, 2009).

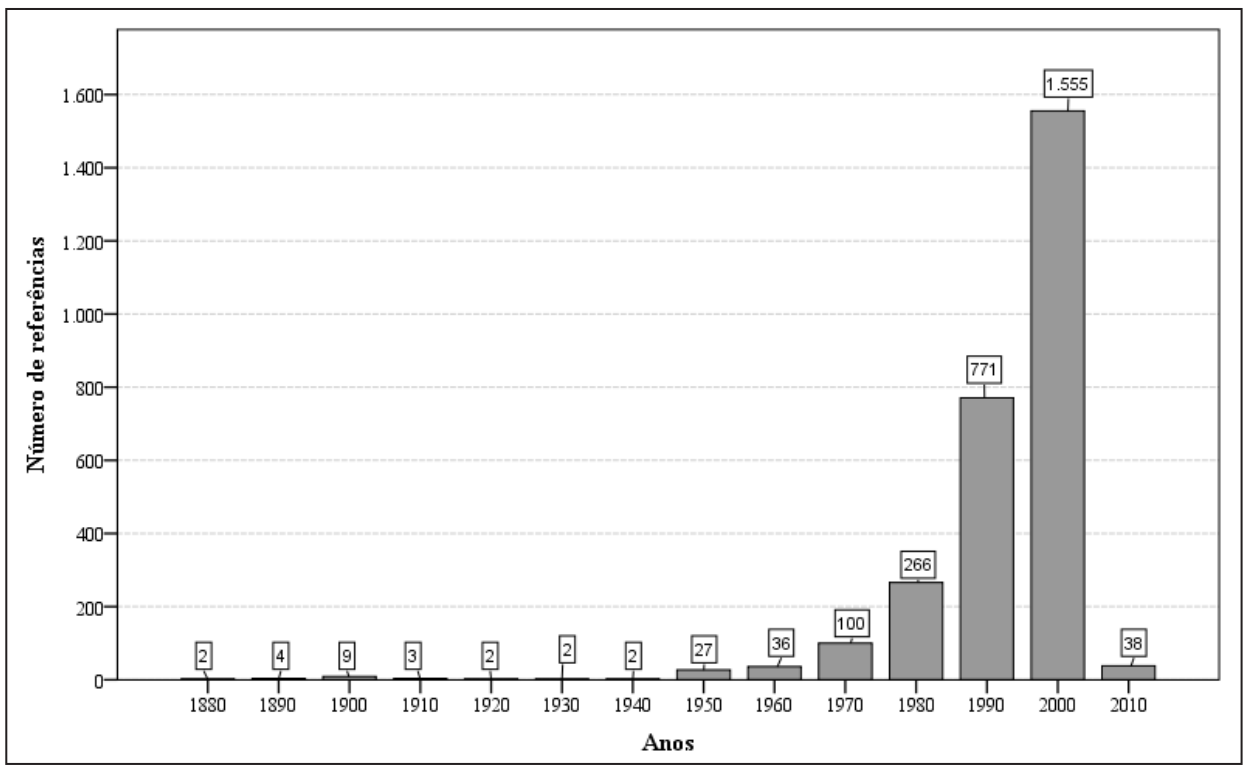

Figura 1. Gráfico de barras da frequência das pesquisas por décadas. 
No que se refere aos dados sobre as publicações nacionais, observou-se uma concentração de autores da região Sul e Sudeste o que corrobora os resultados encontrados por Teixeira e cols. (2007), Rueda (2009) e Melo-Silva, Leal e Fracalozzi (2010). Os autores já haviam afirmado que essas são as regiões que mais publicaram trabalhos científicos na área de OP. A produção advinda de outros estados brasileiros pode estar sendo divulgada em outros veículos, não existir ou ser pouco expressiva. Nessa mesma direção, quanto às universidades dos autores verificou-se que, novamente, a região Sudeste merece destaque no que tange à produção na área. As publicações internacionais no período estudado destacaram Portugal como o país de maior produção, porém há pouca parceria entre os pesquisadores interpaíses, dado identificado também por López e cols. (2010).

Quanto ao tipo de manuscrito, os resultados apontaram para uma maior concentração de publicações de natureza empírica. Esses dados estão em consonância com os achados de Noronha e Ambiel (2006) e Teixeira e cols. (2007), que encontraram nas publicações da década de 1990, em relação às anteriores, um aumento de estudos empíricos em relação aos teóricos. Outros estudos mais recentes como os de Melo-Silva, Leal e Fracalozzi (2010) e Ivatiuk e Yoshida (2010), apontaram para essa mesma realidade, na qual os relatos de pesquisa e de experiências estão em maior número nas publicações.

Os adolescentes são ainda o público mais pesquisado, corroborando estudos anteriores, como o de Teixeira e cols. (2007), por exemplo. Seguramente este é um ponto a ser desenvolvido, qual seja, a diversificação das amostras. A este respeito, o estudo de Ivatiuk e Yoshida (2010) remete à pequena quantidade de investigações com grupos minoritários, o que não está em consonância com a realidade estadunidense retrata por Guindon e Richmond (2005). Os autores, ao analisarem a produção de ano 2004 encontraram trabalhos diversos com grupos distintos, como pessoas com necessidades especiais, pessoas menos favorecidas, dentre outros.

A fundamentação teórica, com exceção dos artigos teóricos, foi pouca abordada. Embora os autores que estão publicando possam supor que a pesquisa empírica não necessite de grande arrazoado teórico, parece que a área de orientação profissional não está isenta da crítica de que a fundamentação precisa ser mais bem discutida, o que já foi abordado por Noronha e Ambiel (2006) e Noronha e cols. (2006). Em grande parte dos artigos observou-se que os autores não deixam explícita a teoria e sua respectiva definição.
A análise das categorias temáticas, quando organizadas em 'abordagem teórica', 'foco no instrumento/ técnica', 'análise de produção cientifica', 'análise de resultados e processos', 'relações entre construtos afins e outras variáveis' e 'outros', revelou que a maior parte das publicações versou sobre as associações entre diferentes conceitos teóricos. Outros estudos que investigaram o estado da arte na área de OP não realizaram tal análise, o que não favorece o diálogo desses resultados com outros (Melo-Silva, 1999; Noronha e cols., 2006; Teixeira e cols., 2007; dentre outros). No entanto, o que se pode afirmar é que, de modo geral, a análise de resultados e processos ainda é pouco priorizada, tal como destacado por Arruda e Melo-Silva (2010), bem como a ênfase no aprimoramento das técnicas e instrumentos de avaliação (Noronha e Ambiel, 2006).

Parte dos manuscritos não apresentou dados referentes à utilização de instrumentos de avaliação. A utilização de testes foi destacada no levantamento de Noronha e Ambiel (2006), que encontraram trabalhos que expressavam a preocupação com a qualidade dos instrumentos de avaliação. Em relação às técnicas utilizadas nos artigos publicados, os resultados mostraram a hegemonia da entrevista como recurso técnico. Esses dados encontrados estão em concordância com os achados de Rueda (2009).

A análise das referências mereceu destaque neste estudo. Duas considerações podem ser feitas em relação ao dado. O levantamento permite identificar o espectro de publicações da área presentes nas publicações. Isto posto, pode-se afirmar que foram citadas referências desde 1880, reafirmando que a área não é recente. Uma segunda observação diz respeito aos períodos preferencialmente referendados nos artigos analisados. Sob esta perspectiva, nota-se que a partir da década de 1970 do século passado, houve aumento de publicações e, consequentemente, de citações. Ademais, ressalta-se que as referências estrangeiras apareceram em maior número, sugerindo maior interesse dos autores em conhecer o que foi publicado fora do contexto nacional.

Por fim, sugere-se que outras pesquisas de avaliação da produção científica investiguem as publicações em orientação profissional oriundas de outros Estados, além do eixo sul e sudeste e também de outros tipos de publicações (livros, bases de dados, outras revistas). Convém destacar que os dados aqui apresentados representam um único periódico científico que divulga a orientação profissional, o que em alguma medida, sugere que os dados sejam generalizados com cautela. A despeito disso, a referida revista tem relevância no cenário brasileiro, por ser a única temática, bem como por sua perspectiva histórica. 


\section{REFERÊNCIAS}

Arbona, C. (2000). Practice and research in career counseling and development - 1999. The Career Developmente Quarterly, 49, 98-134.

Arruda, M. N. F., \& Melo-Silva, L. L. (2010). Avaliação da intervenção de carreira: a perspectiva dos ex-clientes. PsicoUSF, 15(2), 225-234.

Bardin, L. (1977). Análise de conteúdo. São Paulo: Edições 70.

Bufrem, L., \& Prates, Y. (2005). O saber científico registrado e as práticas de mensuração da informação. Ciência da Informação (Brasília), 34(2), 9-25.

Guindon, M. H., \& Richmond, L. J. (2005). Practice and research in career counseling and development -2004 . The Career Development Quarterly, 54(2), 90-137.

Ivatiuk, A. L., \& Yoshida, E. M. P. (2010). Orientação Profissional de pessoas com deficiências: Revisão de literatura (20002009). Revista Brasileira de Orientação Profissional, 11(1), 95-106.

Lehman, Y. P. (2010). Orientação profissional na pós-modernidade. In R. S. Levenfus, \& D. H. P. Soares (Orgs.). Orientação vocacional ocupacional (pp. 19-30). Porto Alegre: Artmed.

Levenfus, R. S. (2010). Orientação vocacional ocupacional: abordagem clínica psicológica. In: R. S. Levenfus, \& D. H. P. Soares (Orgs.). Orientação vocacional ocupacional (pp. 117-132). Porto Alegre: Artmed.

López, W. L., García-Cepero, M. C., Bustamante, M. C. A., Silva, L. M., \& López, E. A. (2010). Panorama General de la Producción Acadêmica en la Psicologia Iberoamericana 2005-2007. Papeles del Psicólogo, 3(3), 296-309.

Melo-Silva, L. L., Leal, M. de S., \& Fracalozzi, N. M. N. (2010). Produção científica em congressos brasileiros de orientação vocacional e profissional: período 1999-2009. Revista Brasileira de Orientação Profissional, 11(1), 107-120.

Noronha, A. P. P., \& Ambiel, R. A. M. (2006). Orientação profissional e vocacional: análise da produção científica. Psico-USF, 11(1), 75-84.

Noronha, A. P. P., Andrade, R. G., Miguel, F. K., Nascimento, M. M., Nunes, M. F. O., Pacanaro, S. V., Ferruzzi, A. H., Sartori, F. A., Takahashi, L. T., \& Cozza, H. F. P. (2006). Análise de teses e dissertações em orientação profissional. Revista Brasileira de Orientação Profissional, 7(2), 1-10.

Noronha, A. P. P., Santos, A. A. A., \& Sisto, F. F. (2010). Contribuições da Escala de Aconselhamento Profissional
(EAP). In R. S. Levenfus, \& D. H. P. Soares (Orgs). Orientação Vocacional Ocupacional (pp. 183-193). Porto Alegre: Artmed.

Rueda, F. J. M. (2009). Produção científica da Revista Brasileira de Orientação Profissional. Revista Brasileira de Orientação Profissional, 10(2), 1-11.

Sabadini, A. A. Z. P., Sampaio, M. I.C., \& Koller, S. H. (Orgs.). (2009). Publicar em Psicologia: um enfoque para a Revista Científica. São Paulo: Casa do Psicólogo.

Sampaio, M. I. C. (2008). Citações a periódicos na produção científica de Psicologia. Psicologia: Ciência e Profissão, 28(3), 452-465.

Teixeira, M. A. P., Lassance, M. C. P., Silva, B. M. B., \& Bardagi, M. P. (2007). Produção científica em orientação profissional: uma análise da revista brasileira de orientação profissional. Revista Brasileira de Orientação Profissional, 8(2), 25-40.

Witter, G. P. (1999). Metaciência e leitura. Em G. P. Witter (Org.). Leitura: textos e pesquisas (pp. 13-22). Campinas: Alínea.

Autores:

Ana Paula porto Noronha - Doutora em Psicologia Ciência e Profissão pela Pontifícia Universidade Católica de Campinas. Docente do Programa de PósGraduação Stricto Sensu em Psicologia da Universidade São Francisco. Bolsista Produtividade em Pesquisa do CNPq.

Cristiane Deantonio Ventura - Aluna do Programa de Pós-Graduação Stricto Sensu em Psicologia da Universidade São Francisco (Mestrado). Bolsistas CAPES.

Dario Cecilio-Fernandes - Aluno do Programa de Pós-Graduação Stricto Sensu em Psicologia da Universidade São Francisco (Mestrado). Bolsista CNPq.

Juliana Carnevalli Siqueira Nery - Aluna do Programa de Pós-Graduação Stricto Sensu em Psicologia da Universidade São Francisco (Mestrado). Bolsistas CAPES.

Juliana Maximila - Aluna do Programa de Pós-Graduação Stricto Sensu em Psicologia da Universidade São Francisco (Mestrado). Bolsistas CAPES.

Luana Luca - Mestre em Psicologia pelo Programa de Pós-Graduação Stricto Sensu em Psicologia da Universidade São Francisco. Bolsista AT/CNPq.

Ivan Baroncelli Neto - Aluno do Programa de Pós-Graduação Stricto Sensu em Psicologia da Universidade São Francisco (Mestrado). Docente da Academia Militar das Agulhas Negras (AMAN).

Maria Áurea Pereira Silva - Mestre em Psicologia Social pela Universidade do Estado do Rio de Janeiro. Aluna do Programa de Pós-Graduação Stricto Sensu em Psicologia Ciência e Profissão da Pontifícia Universidade Católica de Campinas (Doutorado).

\section{Endereço para correspondência:}

Luana Luca

Rua Francisco Glicério, 10, apto 33 - Centro

CEP: 13250-330 Itatiba, SP, Brasil

E-mail: luanagluca@hotmail.com

Recebido em: 17.12.2012.

Aceito em: 19.03.2013. 